\title{
Screening of antibacterial activity in marine green, red and brown macroalgae from the western coast of Libya
}

\author{
Rabia Alghazeer $^{1^{*}}$, Fauzi Whida ${ }^{2}$, Entesar Abduelrhman ${ }^{3}$, Fatiem Gammoudi $^{4}$, Salah Azwai $^{4}$ \\ ${ }^{1}$ Chemistry Department, Faculty of Sciences, Tripoli University, Tripoli, Libya; \\ *Corresponding Author: Rabia_alghazeer@yahoo.com \\ ${ }^{2}$ Botany Department, Faculty of Sciences, Tripoli University, Tripoli, Libya \\ ${ }^{3}$ Biology Department, Faculty of Education, Azzawiya University, Azzawiya, Libya \\ ${ }^{4}$ Microbiology and Parasitology Department, Faculty of Veterinary Medicine, Tripoli University, Tripoli, Libya
}

Received 6 November 2012; revised 10 December 2012; accepted 22 December 2012

\begin{abstract}
Marine macroalgae are considered as an excellent source of bioactive compounds which has a broad range of biological activities including antibacterial and antioxidant. Crude methanolic and water extracts of 19 marine algal species (6 Chlorophyta, 8 Phaeophyta and 5 Rhodophyta) collected from the western coast of Libya were evaluated for antibacterial activity against pathogenic bacteria (4 Gram-positive, 4 Gram-negative). The extracts showed a significant antibacterial activity against Gram positive (Staphylococcus aureus, Bacillus subtilis, Bacillus spp., and Staphylococcus epidermidis) as well as Gram negative bacteria (Escherichia coli, Salmonella typhi, Klebsiella spp., and Pseudomonas aeruginosa). The algal aqueous and methanolic extracts displayed different degrees of antimicrobial activities against different bacteria, in some cases methanolic extracts showed higher antibacterial activity than aqueous extracts. Among tested algae, Brown algae namely Cystoseira crinite exhibited the highest antibacterial activity among tested algal species.
\end{abstract}

Keywords: Macroalgae; Phytochemicals Screening; Antibacterial Activity

\section{INTRODUCTION}

There is an increasing demand for therapeutic drugs from the vastly biodiverse natural resources. The potential contribution of marine organisms to the discovery of new bioactive molecules is remarkably increasing [1-3]. Marine macroalgae are considered as an excellent source of bioactive compounds which has a broad range of bio- logical activities including antibacterial $[4,5]$, antifungal [6], antiviral [5,7], antitumorals [8], antioxidant [9-11], and antiinflammatories [12-14]. Marine macroalgae compose a natural source of a variety of drugs for pharmaceutical, food and cosmetic applications including carotenoids, terpenoids, steroids, amino acids, phlorotannins, phenolic compounds, halogenated ketones, alkanes and cyclic polysulphides $[15,16]$. Therefore, algae have been used in traditional medicine for a long time [17]. Many bioactive compounds of marine algae with antimicrobial activity have been isolated and some of them are under investigation to protect life-style related diseases, some of these compounds are sterols, terpenoids, polysaccharides, peptides, proteins, vitamins, acrylic acid, terpenes, chlorophyllides, phenols, heterocyclic compounds, halogenated ketones and alkanes and cyclic polysulphides [7, 15,18-20]. Libya has a long coastline and abundant natural resources of marine algae with very high species diversity [21], however there are only few reports on the screening of Libyan marine algae for antibacterial activity.

The aim of this study was to assess the antibacterial effect of aqueous and methanolic crude extracts of 19 species of marine algae (6 Chlorophyta, 9 Phaeophyta and 5 Rhodophyta) from the western coast of Libya, against pathogenic bacteria (4 Gram-positive, 4 Gramnegative).

\section{EXPERIMENTAL SECTION}

\subsection{Algae Collection}

Green Algae (Chlorophyta) Ulva lactuca, Enteromorpha compressa, Enteromorpha spp., Enteromorpha prolifera; brown algae (Phaeophyta) Cystoseira barbata, Cystoseira crinita, Cystoseira stricta, Cystoseira compressa, Sargassum vulgare, Dictyopteris membranacea, 
Cladostephus verticillatus, Halopteris filicina, and red algae (Rhodophyta) Gelidium latifolium, Hypnea musciformis, Jania rubens, Jania spp. and Laurencia obtuse, were collected from western coast of Libya between February and March, 2009. The algal samples were authenticated at Botany department, Faculty of Science, Tripoli University. After collection, they were thoroughly washed and then shade dried. The dried samples were crushed in an electric mill till a coarse powder was obtained [22].

\subsection{Bacterial Strains}

Eight bacterial strains (Gram positive and negative) were selected for the study. The Gram positive species were Staphylococcus aureus (S. aur) was obtained from the Clinical Microbiology Laboratory, Azzawiya Medical Center, (Azzawiya, Libya). Bacillus subtilis (B. sub) were kindly provided by Department of Microbiology Biotechnology Research Center (Twaisha), Libya, while $B a-$ cillus spp. (B. spp.) and Staphylococcus epidermidis (S. epi) were obtained from department of Microbiology and Parasytology, Faculty of Veterinary Medicine, Tripoli University. The Gram negative species were Salmonella typhi (S. typhi), Escherichia coli (E. coli), Pseudomonas aeruginosa (P. aer) and Klebsiella spp. (K. spp.), were obtained from the department of Microbiology, Faculty of Veterinary Medicine, Tripoli University, Libya.

\subsection{Preparation of Algae Extracts}

$20 \mathrm{~g}$ of each dried algae powder were extracted with $100 \mathrm{ml}$ of either methanol or distilled water at $100 \mathrm{rpm}$ in a shaker incubator for $24 \mathrm{~h}$ at room temperature (Fedmund Buhler KL2, Germany). The solution was filtered through Whatman No. 1 sterile filter paper. Resulting methanolic extracts were evaporated to dryness using Rotary evaporator (Heidolph 300 LabroRota, Germany), while filtrates of aqueous extracts were dried using freeze dryer. Each dried precipitate was re-dissolved in the corresponding solvents to give $50 \mathrm{mg} / \mathrm{ml}$ extracts, then stored at $-20^{\circ} \mathrm{C}$ until tested.

\subsection{Preliminary Phytochmical Tests}

Preliminary phytochmical tests for identification of alkaloids, anthraquinones, coumarins, flavonoids, saponins, tannins, and terpenes were carried out for all the extracts using standard qualitative methods that have been described previously [23-27].

\subsection{Determination of Antibacterial Activity}

The antimicrobial activity test of algal crude extracts was performed in vitro using the "hole-plate diffusion method" [28]. The respective bacterial culture was poured into the nutrient agar plates for uniform distribution of microorganisms. Using sterile cork borer, $8 \mathrm{~mm}$ wide well was made on each plate. The plates with bacteria were incubated at $37^{\circ} \mathrm{C}$ for $24 \mathrm{~h}$. After incubation, the inhibition zones formed around the holes were measured. Methanol $(100 \%)$ without seaweed extract was used as negative control and Ciprofloxacin disc $(30 \mu \mathrm{g})$ was used as the positive control. All tests were performed in triplicate, and clear zone greater than $10 \mathrm{~mm}$ were considered as positive results [29].

\subsection{Determination of Minimum Inhibitory Concentration (MIC)}

The MICs were determined by the agar dilution method adopted by Daud and Sanchaz (2005) [30]. Two-fold serial dilutions of the original algae extract $(500 \mathrm{mg} / \mathrm{ml})$ were prepared in nutrient broth to obtain concentration from 200 to $12.5 \mathrm{mg} / \mathrm{ml}$ solvent, reference antibiotics and the solvent were also assayed.

\section{STATISTICAL ANALYSIS}

The results for the antibacterial activity was compared by using analysis of variance and Tukey test at $P=0.05$ using statistical software SPSS Windows version 20.

\section{RESULTS AND DISCUSSION}

\subsection{Phytochemical Screening}

The qualitative phytochemical screening of the crude powder of 19 algae was carried out in order to assess the presence of bioactive compounds which might have antibacterial potency. The presence of the alkaloids, flavonoids, tannins, steroids, saponins and anthraquinones was investigated (Table 1). Alkaloids were present in higher amounts $(+++)$ in 3 algae, 10 in moderate amounts $(++)$ and 5 in lower amounts $(+)$ while 1 of tested algae have no alkaloids. Flavanoids were present in 11 algae in higher amounts, 6 in moderate amounts, 1 in low amount and only one showed no presence of flavanoids. Tannins were present in 5 in high amounts, 8 in moderate amounts, 5 in low amounts and only one algae doesn't contain tannins. Presence of flavonoids and alkaloids in most tested algae is interesting because of their possible use as natural additives emerged from a growing tendency to replace synthetic antioxidant and antimicrobials with natural ones [31]. Our results were in agreement with previous findings which showed presence of flavonoids and alkaloids in most of marine algae [32-34].

\subsection{Antibacterial Activity of Algal Extracts}

The inhibitory effects of crude methanol and aqueous extracts of 19 species of Libyan marine algae (green, red, and brown) on the growth of various Gram positive and 
Table 1. Preliminary phytochemical screening of crude extracts of red, brown and green algae.

\begin{tabular}{|c|c|c|c|c|c|c|c|}
\hline & Alkaloids & Tannins & Saponins & Flavonoids & Terpenes & Anthraquinones & Coumarins \\
\hline \multicolumn{8}{|c|}{ Chlorophyta } \\
\hline U. lactuca & ++ & +++ & +++ & +++ & ++ & - & - \\
\hline E. compressa & + & - & + & ++ & + & - & ++ \\
\hline E. prolifera & ++ & ++ & ++ & +++ & ++ & - & + \\
\hline E. spp. & ++ & ++ & +++ & ++ & +++ & + & + \\
\hline C. tomentosum & ++ & +++ & +++ & +++ & + & - & - \\
\hline C. racemosa & +++ & + & +++ & ++ & ++ & - & + \\
\hline \multicolumn{8}{|c|}{ Phaeophyta } \\
\hline C. barbata & +++ & ++ & ++ & +++ & ++ & + & +++ \\
\hline C. crinita & ++ & +++ & + & +++ & ++ & ++ & + \\
\hline C. stricta & + & ++ & + & +++ & +++ & ++ & ++ \\
\hline C. compressa & ++ & ++ & + & ++ & +++ & + & ++ \\
\hline S. vulgare & ++ & ++ & + & +++ & +++ & + & + \\
\hline D. membranacea & ++ & + & + & +++ & +++ & + & - \\
\hline C. verticillatus & + & ++ & +++ & +++ & ++ & - & ++ \\
\hline H. filicina & +++ & + & +++ & +++ & ++ & + & + \\
\hline \multicolumn{8}{|c|}{ Rhodophyta } \\
\hline G. Iatifolium & ++ & ++ & +++ & ++ & +++ & ++ & + \\
\hline H. musciformis & + & + & + & - & - & - & - \\
\hline J. rubens & - & + & ++ & + & +++ & - & ++ \\
\hline$J$. spp. & + & +++ & +++ & +++ & ++ & - & - \\
\hline L. obtusa & ++ & + & ++ & ++ & + & + & +++ \\
\hline
\end{tabular}

+++ve, rich; ++ve, moderate; +ve, poor; -ve, absent.

negative bacteria using agar diffusion method are shown in Tables 2 and 3. The extracts showed a significant antibacterial activity against Gram positive as well as Gram negative bacteria that confirms previous findings $[13,14$, 35]. The algal aqueous and methanolic extracts displayed different degrees of antimicrobial activities against different bacteria, whereas some algae were active against all tested bacteria such as E. compressa, U. lactuca, E. prolifera (green algae) which was in agreement with other reports [36], while others showed no activity against some tested strains.

The Klebsiella spp. was found to be more sensitive (widest zones of inhibition) among the Gram negative bacteria (E. coli, S. typhi, and P. aeruginosa), and $P$. aeruginosa was found to be more resistant. On the other hand, most of tested algae showed inhibitory activity against the tested Gram positive bacteria (B. subtilis, S. epidermidis, S. aureus and Bacillus spp.). In general, the Gram negative bacteria were more resistant (without zones of inhibition), especially for aqueous extracts than the Gram positive bacteria.

Methanol extract of C. racemosa (Chlorophyceae) exhibited strong inhibition against Klebsiella spp. and $S$. typhi with $(16,16 \mathrm{~mm}$ respectively) which was signifi- cantly higher than all other algae extracts $(P<0.05)$, meanwhile, methanol extract of $C$. stricta (Phaeophyceae) showed noticeable activity against $S$. epidermidis and $S$. aureus $(15,15 \mathrm{~mm}$ respectively) in comparison with all algal extracts $(P<0.05)$. G. latifolium (Rhodophyceae) extract showed higher activity against E. coli $(14 \mathrm{~mm})$ than all other algal extracts. The Gram positive B. subtilis strain was more susceptible to ectract of C. racemosa and $C$. stricta extracts (14, $14 \mathrm{~mm}$ respectively) compared to all other methanolic extracts $(P<0.05)$. No inhibitory activities have been observed with some of the methanol or aqueous extracts such as C. tomentosum extact that exhibited no activity against $P$. aeruginosa, $K$. spp. and E. coli (Tables 2 and $\mathbf{3}$ ). In this study, the brown and red algae extracts were found more active than green algae extracts, however brown algal extracts yield higher antibacterial activity than red algae extracts which was in parallel with earlier investigation [37].

Overall, antibacterial activity of aqueous extracts was higher than that of methanol extracts (Table 3). In most cases, aqueous extracts of all algae showed profoundly distinct antibacterial activity by having observable inhibition with diameters ranging from 11 to $18 \mathrm{~mm}$ on tested bacteria. A remarkable effect was obtained with $C$. 
Table 2. Antimicrobial activity of methanolic marine algae extracts. TG = Taxonomic Group (C: Chlorophyta, P: Phaeophyta, R: Rhodophyta).

\begin{tabular}{|c|c|c|c|c|c|c|c|c|c|}
\hline & \multirow{3}{*}{ TG } & \multicolumn{8}{|c|}{ Inhibition Zone $(\mathrm{mm})^{\mathrm{a}}$} \\
\hline & & \multicolumn{4}{|c|}{ Gram positive } & \multicolumn{4}{|c|}{ Gram negative } \\
\hline & & S. epi & S. aur & B. spp. & B. sub & P. aer & K. spp. & S. typhi & E. coli \\
\hline \multirow{10}{*}{$\mathbf{C}$} & U. lactuca & $11 \pm 0.11^{\mathrm{a}}$ & $11 \pm 0.29^{b}$ & $13 \pm 0.58^{b}$ & $11 \pm 0.09^{b}$ & $14 \pm 0.11^{\mathrm{c}}$ & $14 \pm 0.63^{\mathrm{c}}$ & $13 \pm 0.10^{\mathrm{b}}$ & $12 \pm 0.11^{b}$ \\
\hline & E. compressa & $12 \pm 0.21$ & $12 \pm 0.11^{\mathrm{b}}$ & $12 \pm 0.21^{\mathrm{b}}$ & $11 \pm 0.01^{\mathrm{a}}$ & $11 \pm 0.29^{\mathrm{a}}$ & $13 \pm 0.29^{b}$ & $12 \pm 0.09^{b}$ & $12 \pm 0.09^{\mathrm{b}}$ \\
\hline & E. prolifera & $11 \pm 0.01^{\mathrm{a}}$ & $10 \pm 0.11^{\mathrm{a}}$ & $13 \pm 0.08^{\mathrm{b}}$ & $11 \pm 0.09^{b}$ & $12 \pm 0.11^{\mathrm{b}}$ & $10 \pm 0.09^{\mathrm{a}}$ & $11 \pm 0.06^{\mathrm{a}}$ & $11 \pm 0.29^{\mathrm{a}}$ \\
\hline & E. spp. & $12 \pm 0.11^{\mathrm{b}}$ & $14 \pm 0.11^{\mathrm{c}}$ & $14 \pm 1.02^{\mathrm{c}}$ & $13 \pm 0.05^{\mathrm{b}}$ & ND & $15 \pm 0.29^{\mathrm{c}}$ & ND & $11 \pm 0.11$ \\
\hline & C. tomentosum & $13 \pm 0.29^{\mathrm{b}}$ & $13 \pm 0.09^{b}$ & $14 \pm 0.11^{\mathrm{c}}$ & $11 \pm 0.01^{\mathrm{a}}$ & ND & $13 \pm 0.58^{b}$ & $14 \pm 0.29^{\mathrm{c}}$ & ND \\
\hline & C. racemosa & $13 \pm 0.12^{b}$ & $14 \pm 0.29^{c}$ & $13 \pm 0.10^{\mathrm{b}}$ & $14 \pm 0.63^{\mathrm{c}}$ & ND & $16 \pm 1.19^{\mathrm{d}}$ & $16 \pm 0.36^{\mathrm{d}}$ & $12 \pm 0.11^{\mathrm{b}}$ \\
\hline & C. barbata & $11 \pm 0.06^{\mathrm{a}}$ & $13 \pm 0.11^{\mathrm{b}}$ & $14 \pm 0.11^{\mathrm{c}}$ & $11 \pm 0.12^{\mathrm{a}}$ & $11 \pm 0.06^{\mathrm{a}}$ & $15 \pm 0.36^{\mathrm{c}}$ & $11 \pm 0.06^{\mathrm{a}}$ & ND \\
\hline & C. crinita & $12 \pm 0.10^{b}$ & $13 \pm 0.11^{\mathrm{b}}$ & $14 \pm 0.22^{\mathrm{c}}$ & $11 \pm 0.11^{\mathrm{a}}$ & $11 \pm 0.11^{\mathrm{a}}$ & $15 \pm 0.57^{\mathrm{c}}$ & $15 \pm 0.58^{\mathrm{c}}$ & $13 \pm 1.04^{\mathrm{b}}$ \\
\hline & C. stricta & $15 \pm 0.29^{c}$ & $15 \pm 0.29^{c}$ & $13 \pm 0.12^{\mathrm{b}}$ & $14 \pm 0.21^{\mathrm{c}}$ & $12 \pm 0.05^{\mathrm{b}}$ & $11 \pm 0.01^{\mathrm{a}}$ & $15 \pm 0.61^{\mathrm{c}}$ & $12 \pm 0.12^{\mathrm{b}}$ \\
\hline & C. compressa & $12 \pm 0.11^{\mathrm{b}}$ & $13 \pm 0.11^{\mathrm{b}}$ & $12 \pm 0.09^{\mathrm{b}}$ & $\mathrm{ND}$ & $12 \pm 0.07^{\mathrm{b}}$ & $15 \pm 0.09^{c}$ & $13 \pm 0.11^{\mathrm{b}}$ & $13 \pm 0.08^{b}$ \\
\hline \multirow[t]{7}{*}{$\mathbf{P}$} & S. vulgare & $12 \pm 0.12^{\mathrm{a}}$ & $12 \pm 0.10^{\mathrm{b}}$ & ND & $11 \pm 0.01^{\mathrm{a}}$ & $11 \pm 0.01^{\mathrm{a}}$ & $13 \pm 0.11$ & $13 \pm 0.11^{\mathrm{b}}$ & ND \\
\hline & D. membranacea & $14 \pm 0.11^{\mathrm{c}}$ & $14 \pm 0.11^{\mathrm{c}}$ & $11 \pm 0.08^{\mathrm{a}}$ & $12 \pm 0.29^{b}$ & $12 \pm 0.11^{\mathrm{b}}$ & $15 \pm 0.36^{\mathrm{c}}$ & $14 \pm 0.29^{\mathrm{c}}$ & ND \\
\hline & C. verticillatus & $12 \pm 0.09^{\mathrm{b}}$ & $12 \pm 0.11^{\mathrm{b}}$ & $13 \pm 0.46^{\mathrm{b}}$ & $12 \pm 0.11^{\mathrm{b}}$ & $11 \pm 0.09^{\mathrm{a}}$ & $13 \pm 0.11^{\mathrm{b}}$ & $12 \pm 0.07^{\mathrm{b}}$ & ND \\
\hline & H. filicina & $11 \pm 0.06^{\mathrm{a}}$ & $12 \pm 0.13^{\mathrm{b}}$ & $13 \pm 0.05^{\mathrm{b}}$ & $11 \pm 0.09^{\mathrm{a}}$ & ND & ND & $14 \pm 0.40^{\mathrm{c}}$ & $11 \pm 0.12^{\mathrm{a}}$ \\
\hline & G. latifolium & $10 \pm 0.09^{\mathrm{a}}$ & $13 \pm 0.09^{\mathrm{b}}$ & $12 \pm 0.11^{\mathrm{b}}$ & $10 \pm 0.11^{\mathrm{a}}$ & ND & $14 \pm 0.29^{\mathrm{c}}$ & $13 \pm 0.11^{\mathrm{b}}$ & $14 \pm 0.11^{\mathrm{c}}$ \\
\hline & H. musciformis & $11 \pm 0.10^{\mathrm{a}}$ & $11 \pm 0.06^{\mathrm{a}}$ & $12 \pm 0.29^{b}$ & $10 \pm 0.01^{\mathrm{a}}$ & $13 \pm 0.13^{b}$ & $14 \pm 0.34^{\mathrm{c}}$ & $12 \pm 0.13^{\mathrm{b}}$ & $11 \pm 0.09^{\mathrm{a}}$ \\
\hline & J. rubens & $12 \pm 0.06^{b}$ & $11 \pm 0.11^{\mathrm{a}}$ & $12 \pm 0.58^{b}$ & $11 \pm 0.25^{\mathrm{a}}$ & $11 \pm 0.06^{\mathrm{a}}$ & $12 \pm 0.11^{\mathrm{b}}$ & $13 \pm 0.51^{\mathrm{b}}$ & ND \\
\hline \multirow[t]{3}{*}{$\mathbf{R}$} & $J$. spp. & $11 \pm 0.09^{\mathrm{a}}$ & $11 \pm 0.07^{\mathrm{a}}$ & $12 \pm 0.11^{\mathrm{b}}$ & $11 \pm 0.06^{\mathrm{a}}$ & ND & ND & $13 \pm 0.29^{b}$ & $11 \pm 0.21^{\mathrm{a}}$ \\
\hline & L. obtusa & $11 \pm 0.14^{\mathrm{a}}$ & $14 \pm 0.09^{c}$ & $12 \pm 0.09^{b}$ & $11 \pm 0.05^{\mathrm{a}}$ & $12 \pm 0.09^{\mathrm{a}}$ & $13 \pm 0.26^{b}$ & $11 \pm 0.01^{\mathrm{a}}$ & $10 \pm 0.06^{\mathrm{a}}$ \\
\hline & Ciprofloxacin & 23 & 26 & 24 & 20 & 29 & 24 & 25 & 23 \\
\hline
\end{tabular}

S. aur: Staphylococcus aureus B. sub: Bacillus subtilis, E. coli: Escherichia coli, P. aer: Pseudomonas aeruginosa, B. spp.: Bacillus spp., S. typhi: Salmonella typhi, S. epi: Staphylococcus epidermidis, K. spp.: Klebsiella spp. Data are expressed as the mean \pm standard deviation (SD) of three replicates. Different letters represent the statistical comparisons between groups by using ANOVA followed by post hoc Tukey's b test $(P<0.05)$. ND, not detectable.

Table 3. Antimicrobial activity of aqueous marine algae extracts. TG = Taxonomic Group (C: Chlorophyceae, P: Phaeophyceae, R: Rhodophyceae).

\begin{tabular}{|c|c|c|c|c|c|c|c|c|c|}
\hline & \multirow{3}{*}{ TG } & \multicolumn{8}{|c|}{ Inhibition Zone (mm) } \\
\hline & & \multicolumn{4}{|c|}{ Gram positive } & \multicolumn{4}{|c|}{ Gram negative } \\
\hline & & S. epi & S. aur & B. spp. & B. sub & P.aer & K. spp. & S. typhi & E. coli \\
\hline \multirow{10}{*}{$\mathbf{C}$} & U. lactuca & $12 \pm 0.11^{b}$ & $12 \pm 0.08^{\mathrm{a}}$ & $13 \pm 0.11^{b}$ & $12 \pm 0.08^{\mathrm{a}}$ & ND & $11 \pm 0.03^{\mathrm{a}}$ & $11 \pm 0.05^{\mathrm{a}}$ & $12 \pm 0.04^{\mathrm{b}}$ \\
\hline & E. compressa & $15 \pm 0.29^{c}$ & $12 \pm 0.09^{b}$ & $14 \pm 0.13^{\mathrm{c}}$ & $11 \pm 0.01^{\mathrm{a}}$ & $12 \pm 0.29^{b}$ & $15 \pm 0.29^{c}$ & $12 \pm 0.29^{\mathrm{b}}$ & $11 \pm 0.06^{\mathrm{a}}$ \\
\hline & E. prolifera & $11 \pm 0.01^{\mathrm{a}}$ & $12 \pm 0.11^{\mathrm{b}}$ & $11 \pm 0.08^{\mathrm{a}}$ & $11 \pm 0.11^{\mathrm{a}}$ & $11 \pm 0.36^{\mathrm{a}}$ & $11 \pm 0.01^{\mathrm{a}}$ & $11 \pm 0.01^{\mathrm{a}}$ & $11 \pm 0.01^{\mathrm{a}}$ \\
\hline & E. spp. & $12 \pm 0.07^{\mathrm{b}}$ & $11 \pm 0.05^{\mathrm{a}}$ & $16 \pm 0.58^{\mathrm{d}}$ & $11 \pm 0.05^{\mathrm{a}}$ & ND & $12 \pm 0.58^{\mathrm{b}}$ & $12 \pm 0.12^{\mathrm{b}}$ & $11 \pm 0.11^{\mathrm{a}}$ \\
\hline & C. tomentosum & $11 \pm 0.11^{\mathrm{a}}$ & $10 \pm 0.03^{\mathrm{a}}$ & $12 \pm 0.09^{b}$ & $10 \pm 0.01^{\mathrm{a}}$ & ND & ND & $11 \pm 0.01^{\mathrm{a}}$ & ND \\
\hline & C. racemosa & $11 \pm 0.05^{\mathrm{a}}$ & $11 \pm 0.08^{\mathrm{a}}$ & $11 \pm 0.01^{\mathrm{a}}$ & $12 \pm 0.29^{b}$ & ND & ND & $11 \pm 0.02^{\mathrm{a}}$ & ND \\
\hline & C. barbata & $11 \pm 0.11^{\mathrm{a}}$ & -ve & $12 \pm 0.09^{b}$ & $11 \pm 0.05^{\mathrm{a}}$ & ND & ND & $11 \pm 0.03^{\mathrm{a}}$ & ND \\
\hline & C. crinita & $14 \pm 0.29^{c}$ & $15 \pm 0.29^{c}$ & $18 \pm 0.29^{\mathrm{e}}$ & $13 \pm 0.12^{\mathrm{b}}$ & ND & $16 \pm 0.58^{\mathrm{d}}$ & $16 \pm 0.11^{\mathrm{d}}$ & $12 \pm 0.09^{\mathrm{b}}$ \\
\hline & C. stricta & $14 \pm 0.58^{\mathrm{c}}$ & $12 \pm 0.21^{\mathrm{b}}$ & $14 \pm 0.11^{\mathrm{c}}$ & $15 \pm 0.58^{\mathrm{c}}$ & $11 \pm 0.01^{\mathrm{a}}$ & $\mathbf{1 7} \pm 0.23^{\mathrm{d}}$ & $13 \pm 0.06^{\mathrm{b}}$ & $11 \pm 0.01^{\mathrm{a}}$ \\
\hline & C. compressa & $10 \pm 0.01^{\mathrm{a}}$ & $11 \pm 0.01^{\mathrm{a}}$ & $13 \pm 0.11^{\mathrm{b}}$ & $11 \pm 0.05^{\mathrm{a}}$ & $11 \pm 0.03^{\mathrm{a}}$ & $11 \pm 0.01^{\mathrm{a}}$ & $11 \pm 0.03^{\mathrm{a}}$ & $12 \pm 0.29^{b}$ \\
\hline \multirow[t]{7}{*}{$\mathbf{P}$} & S. vulgare & $12 \pm 0.07^{\mathrm{b}}$ & $12 \pm 0.11^{\mathrm{b}}$ & $13 \pm 0.12^{b}$ & $14 \pm 0.36^{\mathrm{c}}$ & $11 \pm 0.01^{\mathrm{a}}$ & $11 \pm 0.11^{\mathrm{a}}$ & $13 \pm 0.08^{b}$ & $11 \pm 0.01^{\mathrm{a}}$ \\
\hline & D. membranacea & $10 \pm 0.02^{\mathrm{a}}$ & $11 \pm 0.01^{\mathrm{a}}$ & $12 \pm 0.02^{\mathrm{b}}$ & $12 \pm 0.11^{\mathrm{b}}$ & ND & $15 \pm 0.05^{\mathrm{c}}$ & $13 \pm 0.08^{b}$ & ND \\
\hline & C. verticillatus & $10 \pm 0.01^{\mathrm{a}}$ & $11 \pm 0.05^{\mathrm{a}}$ & $14 \pm 0.08^{c}$ & $10 \pm 0.01^{\mathrm{a}}$ & ND & $11 \pm 0.03^{\mathrm{a}}$ & $11 \pm 0.01^{\mathrm{a}}$ & $12 \pm 0.05^{\mathrm{b}}$ \\
\hline & H. filicina & $12 \pm 0.08^{\mathrm{b}}$ & $11 \pm 0.04^{\mathrm{a}}$ & $14 \pm 0.13^{\mathrm{c}}$ & $12 \pm 0.09^{b}$ & ND & $14 \pm 0.11^{\mathrm{c}}$ & $14 \pm 0.12^{b}$ & ND \\
\hline & G. latifolium & $15 \pm 0.29^{c}$ & $14 \pm 0.03^{\mathrm{c}}$ & $17 \pm 0.12^{\mathrm{d}}$ & $13 \pm 0.07^{\mathrm{b}}$ & ND & $16 \pm 0.36^{\mathrm{d}}$ & $14 \pm 0.11^{\mathrm{c}}$ & $11 \pm 0.06^{\mathrm{a}}$ \\
\hline & H. musciformis & $15 \pm 0.12^{\mathrm{c}}$ & $12 \pm 0.02^{\mathrm{b}}$ & $16 \pm 0.54^{\mathrm{d}}$ & $11 \pm 0.01^{\mathrm{a}}$ & $12 \pm 0.11^{\mathrm{b}}$ & $15 \pm 0.29^{c}$ & $15 \pm 0.29^{c}$ & $11 \pm 0.03^{\mathrm{a}}$ \\
\hline & J. rubens & $14 \pm 0.23^{\mathrm{c}}$ & $15 \pm 0.09^{c}$ & $15 \pm 0.29^{c}$ & $13 \pm 0.29^{b}$ & ND & $17 \pm 0.58^{\mathrm{d}}$ & $14 \pm 0.11^{\mathrm{c}}$ & $11 \pm 0.01^{\mathrm{a}}$ \\
\hline \multirow[t]{3}{*}{$\mathbf{R}$} & $J$. spp. & $10 \pm 0.01^{\mathrm{a}}$ & $13 \pm 0.21^{\mathrm{b}}$ & $14 \pm 0.11^{\mathrm{c}}$ & $11 \pm 0.03^{\mathrm{a}}$ & ND & $12 \pm 0.12^{\mathrm{b}}$ & $11 \pm 0.05^{\mathrm{a}}$ & $11 \pm 0.01^{\mathrm{a}}$ \\
\hline & L. obtusa & $10 \pm 0.01^{\mathrm{a}}$ & $11 \pm 0.11^{\mathrm{a}}$ & $15 \pm 0.12^{\mathrm{c}}$ & $10 \pm 0.01^{\mathrm{a}}$ & $12 \pm 0.11^{\mathrm{b}}$ & $11 \pm 0.09^{\mathrm{a}}$ & $12 \pm 0.11^{\mathrm{b}}$ & ND \\
\hline & Ciprofloxacin & 23 & 26 & 24 & 20 & 29 & 24 & 25 & 23 \\
\hline
\end{tabular}

S. aur: Staphylococcus aureus B. sub: Bacillus subtilis, E. coli: Escherichia coli, P. aer: Pseudomonas aeruginosa, B. spp.: Bacillus spp., S. typhi: Salmonella typhi, S. epi: Staphylococcus epidermidis, K. spp.: Klebsiella spp. Data are expressed as the mean \pm standard deviation (SD) of three replicates. Different letters represent the statistical comparisons between groups by using ANOVA followed by post hoc Tukey's b test $(P<0.05)$. ND, not detectable. 
crinite (Phaeophyceae) extract against Bacillus spp. (18 $\mathrm{mm})$ and Klebsiella spp. and S. typhi (16, $16 \mathrm{~mm}$ respectively) compared with all other extracts $(P<0.05)$. In addition, $S$. epidermidis was more susceptible to $G$. latifolium, H. musciformis and E. compressa extracts with large high inhibition zone (15 $\mathrm{mm})$.

MIC results for the algal species tested against the different microorganisms are presented in Figures $\mathbf{1}$ and 2. MIC values of $25-200 \mathrm{mg} / \mathrm{ml}$ were obtained for the methanol and aqueous extracts in the tests with the bacterial species. MIC's of the methanol and aqueous extracts for Klebsiella spp. were $25,200 \mathrm{mg} / \mathrm{mL}$ (C. racemosa), 25, $25 \mathrm{mg} / \mathrm{mL}$ (C. crinita), and $50,25 \mathrm{mg} / \mathrm{mL}$ (G. latifolium) respectively. Whereas, MIC's of the methanol and aqueous extracts for Bacillus spp. were 50, 200 $\mathrm{mg} / \mathrm{mL}$ (C. racemosa), 50, $25 \mathrm{mg} / \mathrm{mL}$ (C. crinita), and 100, $25 \mathrm{mg} / \mathrm{mL}$ (G. latifolium) respectively (Figures 1 and 2).

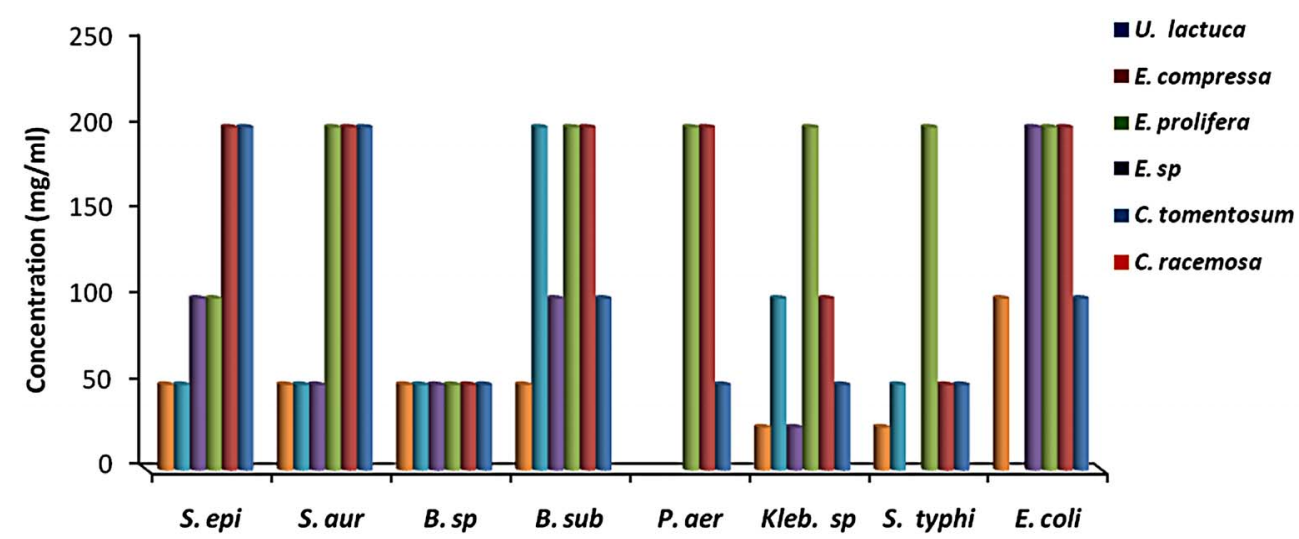

(a)

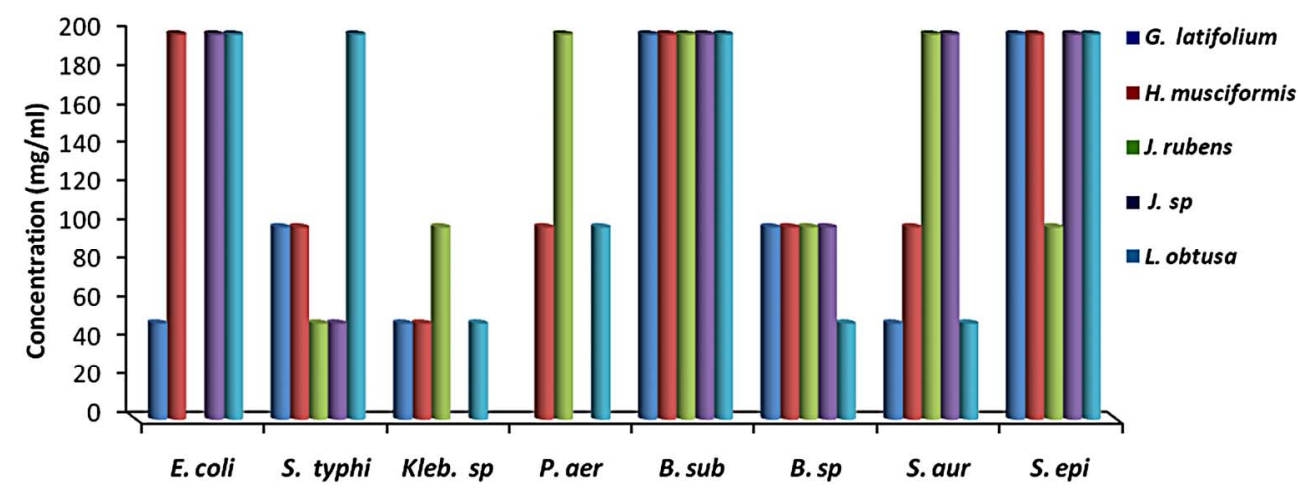

(b)

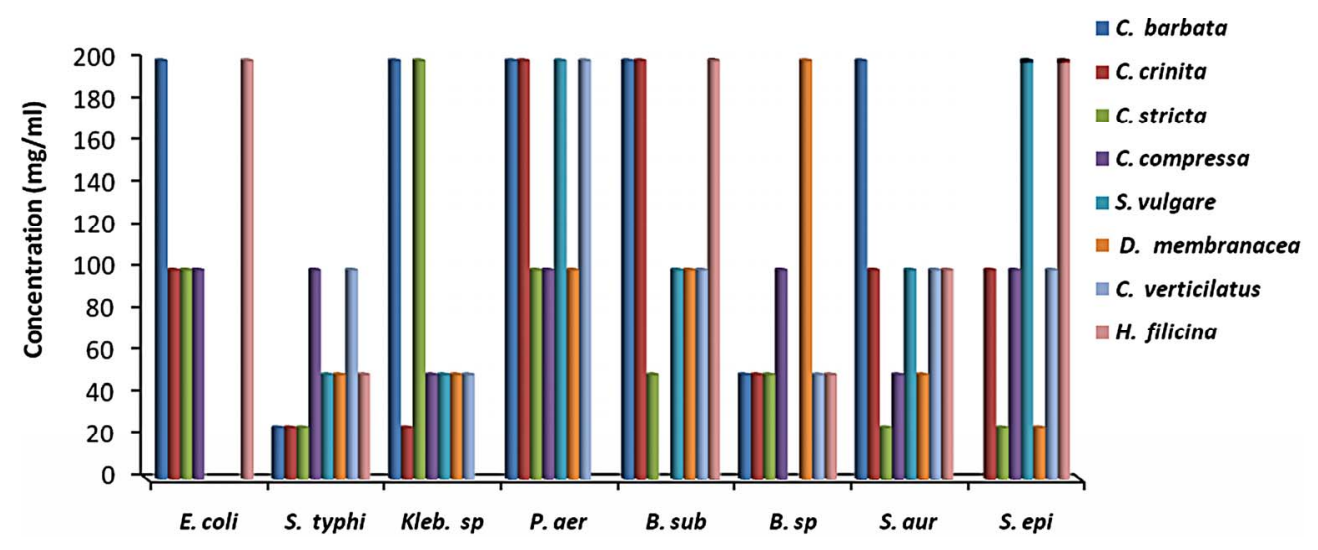

(c)

Figure 1. The in vitro MIC values ( $\mathrm{mg} / \mathrm{ml}$ ) of methanolic extracts of tested algae. (a) Chlorophyta; (b) Phaeophyta; and (c) Rhodophyta. S. aur: Staphylococcus aureus, B. sub: Bacillus subtilis, E. coli: Escherichia coli, P. aer: Pseudomonas aeruginosa, B. spp.: Bacillus spp., S. typhi: Salmonella typhi, S. epi: Staphylococcus epidermidis, K. spp.: Klebsiella spp. 

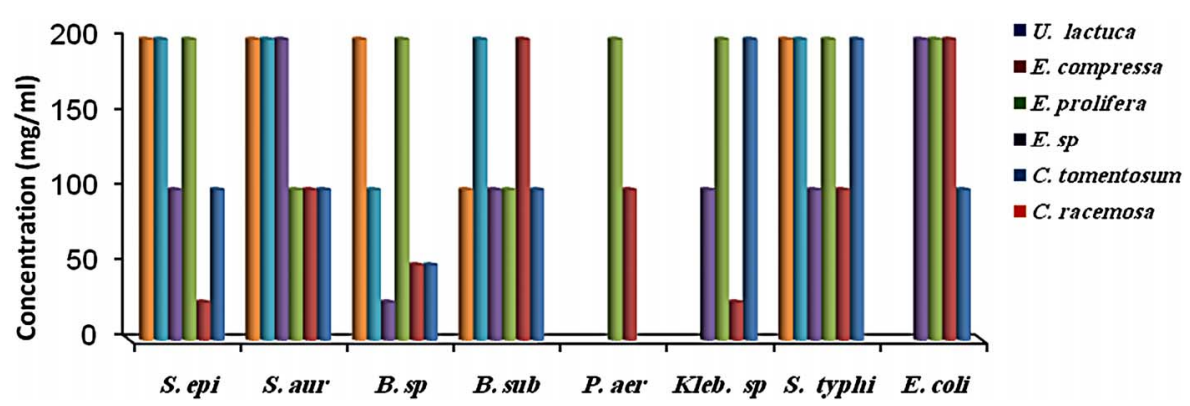

(a)

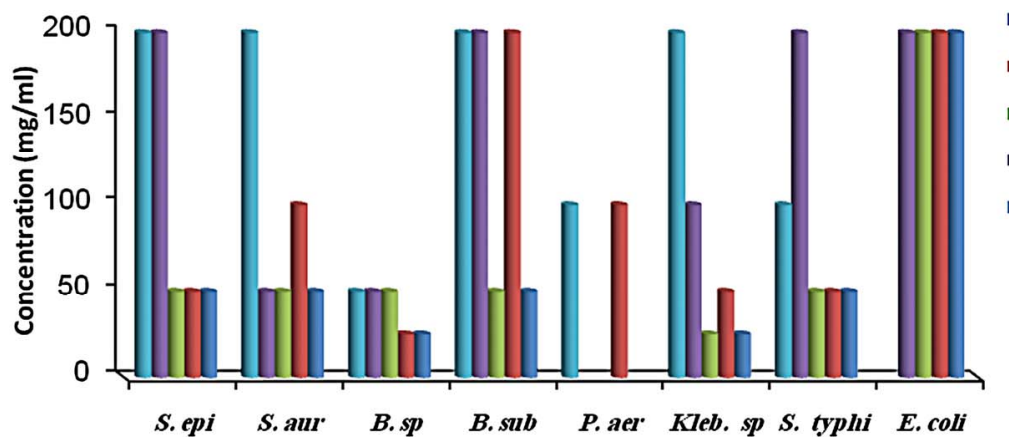

- G. latifolium

- H. musciformis

घ. rubens

- J.sp

$\square$ L. obtusa

(b)

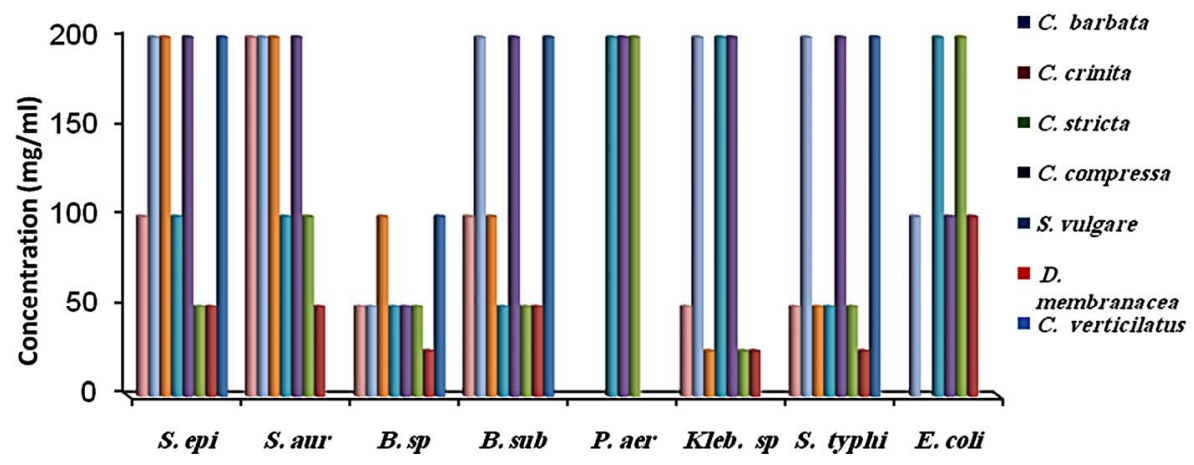

(c)

Figure 2. The in vitro MIC values $(\mathrm{mg} / \mathrm{ml})$ of aqueos extracts of tested algae. (a) Chlorophyta; (b) Phaeophyta; and (c) Rhodophyta. S. aur: Staphylococcus aureus, B. sub: Bacillus subtilis, E. coli: Escherichia coli, P. aer: Pseudomonas aeruginosa B. sp: Bacillus spp., S. typhi: Salmonella typhi, S. epi: Staphylococcus epidermidis, K. spp.: Klebsiella spp.

Most of the active compounds of marine algae show antibacterial activities [36,38]. Many metabolites isolated from marine algae have been shown to possess bioactive effects [39-41]. However, Antimicrobial activity depends on algal species and on extraction efficiency of their active compounds [29], as well as location, seasons of the year and temperature of the water [42]. In present study, methanol extraction from $J$. rubens, $U$. lactuca exhibited antimicrobial activity against $B$. subtilis, whereas their aqueous extracts were inactive (Tables 1 and $\mathbf{2}$ ). In addition, Perez et al. (1990) [43] found that the extract of $U$. lactuca had no antimicrobial activity. In contrast, our results showed that the methanol extract of $U$. lactuca inhibited all the test organisms. This difference may be attributed to location or seasonal variations $[29,44]$.

\section{CONCLUSION}

In conclusion, our results indicate that the algal species collected in the current study from Libyan coast represents rich source of valubal medicine compounds and their extracts exhibit a significant capacity of antibacterial activities especially brown algae, therefore screening their natural products will be of great interest and further studies should be undertaken to characterise the active compounds residing in these types of algae as well as to evaluate the effects of each individual compound on microorganisms. Moreover, toxicological studies are need to be performed. 


\section{ACKNOWLEDGEMENTS}

The authors wish to thank Dr. Hussein, Dr. Amar gelaza and Dr. Asma AlNajjar for their constant encouragement and consultation.

\section{REFERENCES}

[1] Skulberg, O.M. (2000) Microalgal as a source of bioactive molecules-experience from Cyanophyte research. Journal of Applied Phycology, 12, 341-348. doi:10.1023/A:1008140403621

[2] Sithranga, N. and Kathiresan, K. (2010) Anticancer drugs from marine flora: An overview. Journal of Oncology, 21, 41-86. doi:10.1155/2010/214186

[3] Bhatnagar, I. and Kim, S. (2010) Immense essence of excellence: Marine microbial bioactive compounds. Marin Drugs, 8, 2673-2701. doi:10.3390/md8102673

[4] Singh, A. and Chaudhary, B. (2010) Preliminary phycochemical analysis and in vitro antibacterial screening of Pithophora oedogonia (Mont.) Wittrock: A freshwater green alga forming mats in the water bodies. Journal of Algal Biomass Utilization, 1, 33-41.

[5] Bouhlal, R., Riadi, H. and Bourgougnon, N. (2010) Antiviral activity of the extracts of Rhodophyceae from Morocco. African Journal of Biotechnology, 9, 7968-7975.

[6] De Felício, R., Dealbuquerque, S., Young, M.C.M., Yokoya, N.S. and Debonsi, H.M. (2010) Trypanocidal, leishmanicidal and antifungal potential from marine red alga Bostrychia tenella J. Agardh (Rhodomelaceae, Ceramiales). Journal of Pharmaceutical and Biomedical Analysis, 52, 763-769. doi:10.1016/j.jpba.2010.02.018

[7] Bouhlal, R., Haslin, C., Chermann, J.C., Colliec-Jouault, S., Sinquin, C., Simon, G., Cerantola, S., Riadi, H. and Bourgougnon, N. (2011) Antiviral activities of sulfated polysaccharides isolated from Sphaerococcus coronopifolius (Rhodophytha, Gigartinales) and Boergeseniella thuyoides (Rhodophyta, Ceramiales). Marine Drugs, 9, 1187-1209. doi: 10.3390/md9071187

[8] Kim, S.K. and Karadeniz, F. (2011) Anti-HIV activity of extracts and compounds from marine algae. In Advanced Food and Nutrition Research, 64, 255-265. doi:10.1016/B978-0-12-387669-0.00020-X

[9] Kudaa, T., Tsunekawaa, M., Gotoa, H. and Arakib, Y. (2005) Antioxidant properties of four edible algae harvested in the Noto Peninsula, Japan. Journal of Food Composition and Analysis, 18, 625-633. doi:10.1016/j.jfca.2004.06.015

[10] Alghazeer, R., Whida, F., Al-Najjar, A., Majdoob, H. and Al-Mazoghi, E. (2008) Assessment of antioxidant activity and phenolic content of some marine algae from the Libyan Coast. Ain Shams Science Bulletin, 46, 67-78.

[11] Devi, G.K., Manivannan, K., Thirumaran, G., Rajathi, F.A.A. and Anantharaman, P. (2011) In vitro antioxidant activities of selected seaweeds from southeast coast of India. Asian Pacific Journal of Tropical Medicine, 4, 205211. doi:10.1016/S1995-7645(11)60070-9

[12] Vineela, C.H. and Elizabeth, K.M. (2005) Antimicrobial activity of marine algae of Visakhapatnam city, Andhra
Pradesh. Asian Journal of Microbiology, Biotechnology and Environmental Sciences, 7, 209-212.

[13] Tuney, I., Cadirci, B.H., Unal, D. and Sukatar, A. (2006) Antimicrobial activities of the extracts of marine algae from the coast of Urla (Izmir, Turkey). Turkish Journal of Biology, 30, 171-175.

[14] Patra J.K., Rath S.K., Jena, K., Rathod, V.K. and Thatoi, H. (2008) Evaluation of antioxidant and antimicrobial activity of seaweed (Sargassum spp.). Extract: A study on inhibition of glutathione-S-transferase activity. Turkish Journal of Biology, 32, 119-125.

[15] Taskin, E., Ozturk, M., Taskin, E. and Kurt, O. (2007) Antibacterial activities of some marine algae from the Aegean Sea (Turkey). African Journal of Biotechnology, 6, 2746-2751.

[16] Guedes, A., Amaro, M. and Malcata, F. (2011) Microalgae as Sources of Carotenoids. Marine Drugs, 9, 625-644. doi:10.3390/md9040625

[17] Fitton, J.H. (2006) Antiviral properties of marine algae. In: Critchley, A.T., Ohno, M. and Largo, D.B., Eds., World Seaweed Resources, ETI Information Services, Wokingham, 7.

[18] Mtolera, M.S.P. and Semesi, A.K. (1996) Antimicrobial activity of extracts from six green algae from Tanzania. In: Björk, M., Semesi, A.K., Pedersén, M. and Bergman, B., Eds., Current Trends in Marine Botanical Research in the East African Region, SIDA, 211-217.

[19] Bhacuni, D.S. and Rawat, D.S. (2005) Bioactive marine natural products. Springer, New York, 400.

[20] Priyadharshini, S., Bragadeeswaran, S., Prabhu, K. and Ran, S.S. (2011) Antimicrobial and hemolytic activity of seaweed extracts Ulva fasciata (Delile 1813) from Mandapam, Southeast coast of India. Asian Pacific Journal of Tropical Biomedicine, 1, S38-S39. doi:10.1016/S2221-1691(11)60118-4

[21] Nizamuddin, M., West, J.A. and Meñez, E.G. (1979) A list of marine algae from Libya. Botanica Marina, 22, 465-476. doi:10.1515/botm.1979.22.7.465

[22] Chiheb, I., Riadi, H., Martinez-Lopez, J., Dominguez, S., Gomez, V.J., Bouziane, H. and Kadiri, M. (2009) Screening of antibacterial activity in marine green and brown macroalgae from the coast of Morocco. African Journal of Biotechnology, 8, 1258-1262.

[23] Fadeyi, M.G., Adeoye, A.E. and Olowokodejo, J.D. (1989) Epidermal and phytochemical studies with genus of Boerhavia (Nyetanginaceae). International Journal of Crude Drug Research, 29, 178-184.

[24] Odebiyi, A. and Sofowora, A.E. (1990) Phytochemical screening of nigerian medicinal plants. Part III. Lloydia, 41, 234-246.

[25] Harborne, J.B. (1992) Phytochemical methods. Chapman and Hall Publications, London, 7-8.

[26] Abulude, F.O., Onibon, V.O. and Oluwatoba, F. (2004) Nutrition and nutritional composition of some tree barks. Nigerian Journal of Basic and Applied Sciences, 13, 4349.

[27] Abulude, F.O. (2007) Phytochemical screening and mineral contents of leaves of some Nigerian woody plants. 
Research Journal of Phytochemistry, 1, 33-39. doi:10.3923/rjphyto.2007.33.39

[28] Manilal, A., Sujith, S., Kiran, G.S., Selvin, J., Shakir, C., Gandhimathi, R. and Panikkar, M.V.N. (2009) Biopotentials of seaweeds collected from southwest coast of India. Journal of Marine Science and Technology, 17, 67-73.

[29] Lima-Filho, M., Carvalho, U. and Freitas, M. (2002) Antibacterial activity of extracts of six Macroalgae from the Northeastern Brazilian Coast. Brazilian Journal of Microbiology, 33, 311-313. doi:10.1590/S1517-83822002000400006

[30] Daud, A., Gallo, A. and Sanchez, A. (2005) Antimicrobial properties of Phrygilanthus acutifolius. Journal of Ethnopharmacology, 99, 193-197. doi:10.1016/j.jep.2005.01.043

[31] Shan, B., Cai, Y.Z., Brooks, J.D. and Corke, H. (2007) The in vitro antibacterial activity of dietary spice and medicinal herb. International Journal of Food Microbiology, 117, 112-119. doi:10.1016/j.ijfoodmicro.2007.03.003

[32] Wang, C., Mingyan, W., Jingyu, S., Li, D. and Longmei, Z. (1998) Research on the chemical constituents of Acanthophora spicifera in the South China. Bopuxue Zazhi, 15, 237-242.

[33] Zeng, L.-M., Wang, C.-J., Su, J.-Y., Du, L., Owen, N. L., Lu, Y., Lu, N. and Zheng, Q.-T. (2001) Flavonoids from the red alga Acanthophora spicifera. Chinese Journal of Chemistry, 19, 1097-1100. doi:10.1002/cjoc. 20010191116

[34] Güven, K., Percot, A. and Sezik, E. (2010) Alkaloids in marine algae. Marine Drugs, 8, 269-284. doi:10.3390/md8020269

[35] Martin, R.F., Ramos, M.F., Herfibdal, L., Sousa, J.A., Skaerven, K. and Vasconcelos, V.M. (2008) Antimicrobial and cytotoxic assessment of marine cyanobacteria: Synechocystis and Synechococcus. Marine Drugs, 6, 1-11. doi:10.3390/md6010001

[36] Del Val, A.G., Platas, G., Basilio, A., Cabello, A., Gorrochateui, J., Suay, I., Vicente, F., Portillo, E., DeRio, M.J., Reina, G.G. and Pelaez, F. (2001) Screening of antimicro- bial activities in red, green and brown macroalgae from Gran Canaria (Canary Islands, Spain). International Microbiology, 4, 35-40.

[37] Lavanya, R. and Veerappan, N. (2011) Antibacterial potential of six seaweeds collected from gulf of mannar of southeast coast of India. Advances in Biological Research, 5, 38-44.

[38] Vairappan, C.S., Daitoh, M., Suzuki, M., Abe, T. and Masuda, M. (2001) Antibacterial halogenated metabites from the Malysianlaurencia species. Phytochemistry, 58, 291297. doi:10.1016/S0031-9422(01)00243-6

[39] Oh, K.B., Lee, J., Chung, S., Shin, J., Shin, H.J., Kim, H.K. and Lee, H.S. (2008) Antimicrobial activities of the bromophenols from the red alga Odonthalia corymbifera and some synthetic derivatives. Bioorganic and Medicinal Chemistry Letters, 18, 104-108.

[40] Venkateswarlu, S., Panchagnula, G.K., Gottumukkala, A.L. and Subbaraju, G.V. (2007) Synthesis, structural revision, and biological activities of 4'-chloroaurone, a metabolite of marine brown alga Spatoglossum variabile. Tetrahedron, 63, 6909-6914. doi:10.1016/i.tet.2007.04.048

[41] Yang, L., Peng, L.S. and Zhou, S. (2006) Lactones from a brown alga endophytic fungus (No. ZZF36) from the South China Sea and their antimicrobial activities. Bioorganic and Medicinal Chemistry Letters, 16, 4205-4208. doi:10.1016/j.bmcl.2006.05.081

[42] Febles, C.I., Arias, A., Hardisson, A. and Lopez, A.S. (1995) Antimicrobial activity of extracts from Canary species of Phaeophyta and Chlorophyta. Phytotherapy Resarch, 9, 385-387. doi:10.1002/ptr.2650090517

[43] Perez, R.M., Avila, J.G. and Perez, G. (1990) Antimicrobial activity of some American algae. Journal of Ethnopharmacology, 29, 111-118. doi:10.1016/0378-8741(90)90104-2

[44] Moreau, J., Pesando, D. and Bernad, P. (1988) Seasonal variations in the production of antifungal substances by some Dictyotales (brown algae) from French Mediterranean coast. Hydrobiology, 162, 157-162. doi:10.1007/BF00014538 